the treatment of affine spaces and the discussion of hyperplanes, orientation and Grassmannians.

The second part, comprising chapters 9-14, is an introduction to geometric algebra, beginning with Euclidean spaces and bilinear forms. Then follows a discussion of the geometry of the quaternions, correlations, and correlated spaces, which culminates in the definitions of the classical Lie groups and the interpretation of Grassmannians and quadrics as homogeneous spaces. Finally, there is a good exposition of Clifford algebras and Cayley algebra.

The last six chapters present an introduction to differential calculus in Banach spaces, leading up to the definition of differentiable manifolds. They begin with the elements of normed linear spaces, topological spaces, topological groups and topological manifolds. Then differentiable maps are defined for Banach spaces, their properties are studied, and the inverse and implicit function theorems are proved. This is then used as the basis for a brief introduction to smooth manifolds, tangent bundles, embedding theorems, Lie groups and Lie algebras.

The book is well set out with a good index and a list of symbols used, (some of which, unfortunately, are nonstandard). It has plenty of examples and there is a wealth of problems at the end of each chapter. By way of criticism, however, it should be said that the first section is more than amply covered in existing texts, and it might have been better to have assumed a knowledge of the material covered there in order to produce a book which, in paperback at least, might have had a price much more attractive to the undergraduate. It is also debatable whether the material covered in the second section is wholly suitable for an undergraduate course. Nevertheless, the book should prove to be very profitable prerequisite reading for an MSc course in topology or geometry.

$$
\text { L. M. WOODWARD }
$$

\section{Markov Chains}

Discrete Markov Chains. By V. I. Romanovsky, Translated from the Russian by E. Seneta. Pp. xi+408. (Wolters-Noordhoff: Gröningen, The Netherlands, 1970.) Hlf.66,85; \$18.65.

Vsevolod IVANOVICH ROMANOVSKY (1879-1954) was one of the founding fathers of mathematical statistics in the Soviet Union. Born at Alma Ata, he studied at St Petersburg University, presumably under Markov, but returned to Central Asia, where he spent most of his working life, and built up a small but active school in probability and mathematical statistics. During the 20 s and $30 \mathrm{~s}$ he wrote a large number of articles on a variety of topics in mathematical statistics, including several textbooks. Markov chains formed a major interest in the later part of his life, and the present book, the first of his books to be translated into English, contains a systematic account of his work on this topic.

In essence, it is a treatise on the application of classical matrix theory to a variety of problems in finite Markov chains. Much of the basic material overlaps with the monograph by Fréchet ${ }^{1}$, or is subsumed by later work such as the texts by Feller ${ }^{2}$ or Chung ${ }^{3}$. Of the more specialized material a long chapter is devoted to correlations and asymptotic properties of the numbers of visits to the different states. The basic tool here is the method of characteristic functions, which leads to the study of the matrix iterates $P(\theta)^{n}, P(\theta)_{i j}=p_{i j} e^{i \theta} j$. In the following chapters this technique is extended to the study of sums of random variables defined on the Markov chain, to more general bivariate chains $\left(X_{n} Y_{n}\right)$. This work of Romanovsky's, which dates back to a paper in 1939, and includes versions of a central limit theorem for all types of processes discussed, seems to have been overlooked by later writers who have analysed such processes in more general cases, though the underlying ideas are similar, and indeed can be traced to Markov's original memoirs. Another novel feature is the long chapter on cyclic chains, which are interpreted broadly to include not only periodic chains, in the usual sense, but also "bicyclic" and "polycyclic" chains, where there are several cycles of states with some group or groups of states in common.

In summary, it is a book of uneven quality, cumbersome and repetitious in many places, superseded in others, overlapping to a large degree with better and more recent books. Nevertheless, it contains ideas which have not been explored in detail elsewhere, and, at least for Western readers, it provides an interesting and hitherto inaccessible link in the Markov chain story. The translation is good, and a small bibliography of more recent work is provided. It is not suitable for a textbook, but as a collector's item is worthy of attention from any serious student of Markov chains.

D. VERE-JONES

${ }^{1}$ Fréchet, M., Théorie des Evénements en Chaine dans le cas d'un Nombre Fini d'états Possible (Paris, 1938).

${ }^{2}$ Feller, W., Introduction to Probability Theory and its Applications, 1 (New York, 1949).

${ }^{3}$ Chung, K. L., Markov Processes with Stationary Transition Probabilities (Berlin, 1960).

\section{Neutron Diffraction}

Theory of Thermal Neutron Scattering: The Use of Neutrons for the Investigation of Condensed Matter. By W. Marshall and S. W. Lovesey. Pp. xxiii+ 599. (Clarendon: Oxford; Oxford University: London, September 1971.) $£ 12$.

EXPERIMENTERS in neutron diffraction have from classical times been buoyed up by the rumour that Dr Marshall was writing a book on their subject. Now that the advent season is over, after several false starts, it is not surprising that the product should be looked at with a critical eye and we must be careful not to examine the book from the point of view of what it does not achieve rather than what it does.

The intention of the book is clearly stated in the preface as "to give a comprehensive review of all the theory of thermal neutron scattering that is relevant to the interpretation of experiment" and this it does in a masterly way, but I hope to be forgiven for commenting that, in the main, and there are some exceptions, the interpretation is being carried out by a theorist, whereas I had hoped to find Dr Marshall encouraging the experimenter to operate on a do-it-yourself basis.

Bearing in mind that this is the first book to attempt to bring together in a comprehensive way the intense activity of the past twenty years, interpreting the use of neutron beams for studying solids and liquids, the depth of the treatment is quite remarkable. It would be conveyed scarcely at all by the chapter headings, for these are conventional enough-with the familiar "Nuclear Bragg Scattering" and "Neutron Scattering from Liquids" among them. The overall span of the work can be realized by noting that the topics conjured up by the words nuclear, Bragg, phonons, magnetic, spin-waves, polarization, liquids, gases, critical and defects all receive extensive treatment in rigid, quantitative fashion. A better idea of the depth is given by the fact that the introductory "List of Important Symbols" extends to 258 items and the number of equations judged to be sufficiently important to be identified numerically for subsequent reference is 2,013 .

No one who is actively working in the neutron beam field can afford to be without this book or reasonably be ungrateful to the authors for making this immense contribution. The authors make a conscious decision not, in general, to quote the original references to all the ideas and observations which they discuss. This has advantages and disadvantages, and it certainly lessens the value of the author index. A grumble may be justified here, for it seems to be a matter of chance whether an author named in the text is listed.

G. E. BACON 